\title{
Strategies to deal with comorbid physical illness in psychosis
}

\author{
M. Docherty ${ }^{1,2}$, B. Stubbs ${ }^{3,4}$ and F. Gaughran ${ }^{1,5,6 *}$ \\ ${ }^{1}$ National Psychosis Service, South London and Maudsley NHS Foundation Trust, Denmark Hill, London SE5 8AZ, UK \\ ${ }^{2}$ Institute of Psychiatry, Psychology and Neuroscience, Kings College London, De Crespigny Park, London SE5 8AF, UK \\ ${ }^{3}$ Physiotherapy Department, South London and Maudsley NHS Foundation Trust, Denmark Hill, London SE5 8AZ, UK \\ ${ }^{4}$ Health Service and Population Research Department, Institute of Psychiatry, King's College London, De Crespigny Park, London SE5 8AF, UK \\ ${ }^{5}$ Institute of Psychiatry, Psychology and Neuroscience, Kings College London, De Crespigny Park, London SE5 8AF, UK \\ ${ }^{6}$ Collaborative Leadership in Applied Heath Research Centre and Care, South London, UK
}

\begin{abstract}
Individuals with serious mental illnesses such as psychosis still experience higher mortality rates than the general population, decades after data have linked the gap to increased rates of physical illness, delayed diagnosis, low treatment rates and worse outcomes from treatment received. The nature of the relationship between psychosis and comorbid physical illness is complex. Multiple strategies directed at different levels of disease process, health care systems and stakeholder culture are likely required to make sustained progress in reducing the mortality gap. Evidence for strategies that effectively reduce the burden of physical co-morbidity and lead to improved health outcomes are still in their infancy but growing at a reassuringly fast rate. This editorial considers the existing evidence base and makes suggestions for the development and future direction of this urgent research agenda and how this knowledge can be implemented in clinical practice.
\end{abstract}

First published online 18 February 2016

Key words: Cardiometabolic, physical illness, psychosis, serious mental illness.

\section{Physical illness and psychosis: the mortality and morbidity gap}

The mortality gap between the general population and those with serious mental illness (SMI) such as psychosis has been known since the 1800s (Oslo, 1936; Harris \& Barraclough, 1998; Singer, 2001). Some of the greatest reductions in life expectancy are seen in people with schizophrenia. A recent study in South London demonstrated that men with schizophrenia and women with schizoaffective disorder experience a reduced life expectancy of 14.6 and 17.5 years, respectively (Chang et al. 2011). The problem is a global one, with the most recent meta-analysis demonstrating that mortality rates are elevated across all continents (Walker et al. 2015).

Significant contributors to this excess mortality primarily include physical health conditions (Fleischhacker et al. 2008; Tiihonen et al. 2009; De Hert et al. 2012). Whilst suicide rates remain relatively stagnant in this population, deaths attributed to physical comorbidities and in particular cardiometabolic and cardiovascular disease (CVD) are increasing and account for the largest

\footnotetext{
* Address for correspondence: Dr F. Gaughran, Institute of Psychiatry, Psychology and Neuroscience, Kings College London, De Crespigny Park, London SE5 8AF, UK.

(Email: fiona.p.gaughran@kcl.ac.uk)
}

portion of premature deaths (Lawrence et al. 2013; Correll et al. 2015). Risk factors contributing to the CVD burden include significantly higher rates of smoking, obesity, hypertension, metabolic syndrome, diabetes and dyslipidaemia (Osborn et al. 2006). The aggregate effect of these risk factors is a mortality rate from cardiac deaths twice that of the general population (Lawrence et al. 2013; Walker et al. 2015).

Despite the higher prevalence of co-morbid physical illness there is significant under diagnosis (Lawrence \& Kisely, 2010; Smith et al. 2013; Moore et al. 2015), poorer access to preventative interventions such as screening programmes (Druss et al. 2001; Mitchell et al. 2014), lower treatment rates (Druss et al. 2001; Nasrallah et al. 2006; Kisely et al. 2009; Laursen et al. 2009; Mitchell et al. 2009; Scott et al. 2012) and also poorer clinical outcomes (Kisely et al. 2005; Lawrence \& Kisely, 2010; Crawford et al. 2014) relative to the general population.

The association of poorer physical health in SMI can add significantly to the experience of reduced quality of life, disability and more severe symptoms of mental illness (Dixon et al. 1999, 2001). It can compound the existing risk of social exclusion associated with SMI by magnifying pre existing inequalities such as poverty.

The arguments from equity for a need to address this disparity are well rehearsed (Comission, 2005; Fleischhacker et al. 2008; Thornicroft, 2011). Data lend credence to the denotation of this problem as a 
'Scandal' revealing many current public health interventions and physical health care-pathways as inadequate to meet the needs of those with SMI. It has been argued that this should be viewed not solely as a clinical challenge but as more alarming evidence of systematic discrimination and a contravention of the international convention for the Right to Health (Thornicroft, 2011).

Frameworks to address aspects of this problem are now well established from disease management perspectives with multiple existing guidelines on the prevention and management of CVD and its risk factors (e.g., (ADA, 2004; De Hert et al. 2009; NICE, 2014)), an increasing number of policy statements provide a mandate to address the mortality and morbidity gap as part of an agenda to achieve Parity of Esteem (MaP, 2009; RCPsych, 2009, 2013). Despite these tools and the force of now established arguments for active change, the pace of development, evaluation and implementation of evidence-based strategies including service re-configurations to address the problem has been relatively slow.

Delays in achieving progress in this area reveal the complexity of the problem itself, of the systems in which it needs to be mediated and of the barriers within these systems. There are complex relationships between SMI, poor physical health, premature mortality and current health systems which encompass not only care pathways, but also culture and attitudes embedded within them.

Despite growing consensus as to the importance of this problem, a real change in outcomes will not be generated without clear ownership, leadership and delineation of roles and responsibilities between stakeholders within the mental and physical health communities. For four decades there have been repeated calls for Mental Health to take primary responsibility for the overall care of their patients (Wilkinson, 1979; Lamb, 1989; Shore, 1996) without clear impact on changes in clinical practice (Lawrence \& Kisely, 2010). At present the existing evidence base is inadequate to mediate the on-going differing opinion as to the best place to deliver basic primary and secondary prevention for conditions such as cardiometabolic disease. The key stakeholders in this debate however are service users and carers. Their role in the design of integrated services, generating the evidence base for interventions and directly inputting into programmes for peer support and education is paramount if parity of access to physical health care and improved health outcomes are to be achieved.

Ambiguity around ownership of the problem is compounded also by stigma. This impacts not only on the expectations and subsequent efforts of mental and physical health providers to deliver effective interventions, but also on those with SMI in seeking them. Empowering, supporting and offering education to service users to help them make informed decisions about lifestyle choices that impact on physical health is the foundational step in addressing this issue. Several factors such as media representation of mental illness and trends towards more defensive clinical practice may have contributed to a professional culture and practice that has placed disproportionate emphasis on risk domains of homicide and suicide and overshadowed awareness of risk to health from physical illness. These issues place an onus on mental health services to show leadership in forging collaborations with physical health providers and to advocate for change.

\section{Strategies to manage physical illness in psychosis}

Strategies to manage co-morbid physical illness need a range of targets encompassing the disease process and the systems in which health care is delivered (Lambert et al. 2003; Druss, 2007). Successful strategies will require sensitivity to the larger context. Targets include disease-specific primary, secondary and tertiary preventions, interactions between the health care systems in which they are delivered and the health care workers delivering them.

\section{Health promotion and primary prevention}

A promising research body is developing focusing on primary prevention through health promotion programmes and reduction of cardiometabolic risk factors (Shiers et al. 2009). Focus on this area is a priority. There is a significantly lower cardiovascular risk in the earlier stages of schizophrenia than in its later stages. Diabetes and pre-diabetes is relatively uncommon in the early stages of schizophrenia, particularly in unmedicated individuals (Mitchell et al. 2013). Weight and metabolic changes are more difficult to reverse when established (Mitchell \& De Hert, 2015) and disease prevention strategies are generally more cost-effective with greater longitudinal benefits.

It is unlikely however that general public health campaigns significantly influence lifestyle choices of those with psychosis. Whilst public health initiatives such as smoking cessation campaigns have been successful in driving down smoking rates in the general population, relatively little progress has been made among people with SMI with the smoking gap continuing to widen (Cook et al. 2014). In respect of impact from population health messages, Mitchell et al. (2014) similarly demonstrated that mammogram screening rates are significantly reduced among people with SMI. These observations suggest that targeted patient specific and tailored interventions are required. 
A number of non-pharmacological interventions for individuals with SMI and cardiometabolic risk factors have been investigated. These strategies have focused on exercise, diet and overall healthy lifestyle incorporating aspects such as motivational interviewing and cognitive behavioural therapy techniques. There is good reason to be optimistic. A previous meta-analysis by Caemmerer et al. (2012) found across 17 studies that behavioural non-pharmacological interventions led to a significant reduction in weight $(-3.12 \mathrm{~kg}$; CI: -4.03 , $-2.21 ; p<0.0001)$ and body mass index (BMI) $(-0.94$ $\mathrm{kg} / \mathrm{m}^{2} ; \mathrm{CI}:-1.45,-0.43 ; p=0.0003$ ) compared with control groups. The benefits extended to improvements in waist circumference, per cent body fat, glucose, insulin, total cholesterol, low-density -lipoprotein-cholesterol and triglycerides. More recently, Rosenbaum et al. (2014) found that physical activity interventions can improve psychopathological symptoms and to a lesser degree anthropometric measures including weight loss. A number of very well-resourced randomised controlled trials (e.g., Daumit et al. 2013; Rubin et al. 2013) have demonstrated that weight loss although challenging, is possible in those with established illness. Cardiorespiratory fitness is a better predictor of mortality than BMI (Barry et al. 2014) and can improve with exercise, independent from changes in BMI (Vancampfort et al. 2015a). This highlights the relevance of strategies directed at both parameters.

In respect of replicating and implementing such interventions, evidence is available to guide service planners as to the most optimal models of care. A recent qualitative review of reviews of non-pharmacological interventions addressing obesity in SMI (Ward et al. 2015) concluded that personalised interventions with multiple components, more frequent face-to-face contact, and adequately trained treatment providers are associated with the best outcomes. Exercise programmes delivered by qualified exercise personnel (Physiotherapists, exercise physiologists or those with BSc level qualification) result in significantly fewer dropouts than when delivered by non-qualified staff (Vancampfort et al. 2015c). This raises an important point for funders as it suggests that investment is required to achieve optimal outcomes. The transferability of these trials to severely resource limited services is however questionable and rigorous cost effectiveness analyses and follow up data on sustainability of outcomes are required.

\section{The role of mental health services}

The implementation of health promotion programmes must be preceded by a unified effort from Mental Health Professionals to reduce iatrogenic contribution to cardiometabolic risk. Given the relationship between psychotropic medication and metabolic side effects, judicious and individualised prescribing throughout the illness, particularly at its early stages (Mitchell et al. 2013) is essential. In the UK, recent NICE guidelines (NICE, 2014) have specified detailed requirements for monitoring and cardiovascular risk interventions and designated mental health providers as responsible for meeting these requirements. Systems to enforce or incentivise implementation are likely required (Citrome \& Yeomans, 2005). An equally focused approach should be implemented globally in child and adolescent services given the high transition rates to adult services.

Strategies to improve prescribing and disseminate up to date evidence on the relative metabolic risk factors of different antipsychotic medications (Vancampfort et al. $2015 b$ ) or augmentation regimes that may assist in weight loss, should be supported by regulatory bodies and incorporated into guidelines and formularies. Studies exploring the efficacy and cost-effectiveness of augmentation strategies such as metformin or aripiprazole (Wu et al. 2008; Fleischhacker et al. 2010) should be extended. Likewise, careful work is required on the question of switching anti-psychotics to improve cardiometabolic profile. The evidence at present does not suggest that this is an effective strategy at a generic level (Bak et al. 2014), although it may have potential for a given treatment-responsive individual.

Awareness of the impact of psychotropic medication on cardiometabolic health is essential but trading better physical health for poorer mental health should not become an inevitable outcome. Good mental health symptom control is paramount to an individual's ability to mange their physical health (Dixon et al. 1999). Individualised and tailored prescriptions that comprise a package of medication, education and personalised support for physical health should be fully trialled before switching to psychotropic medications that are less efficacious for that individual. Despite clear guidance as to the benefit of clozapine in refractory illness, underprescribing may be in part due to its high metabolic side effects (De Hert et al. 2011). Good evidence that clozapine contributes to increased life expectancy despite this profile highlights the absolute importance of addressing mental health symptoms and working intensively with side effects rather than providing suboptimal mental health treatment (Tiihonen et al. 2009).

In addition to blanket monitoring and screening, an argument can be made for the role of risk stratification and targeting of resources to high-risk groups (Mitchell et al. 2013). Those requiring polypharmacy or high potency antipsychotics should trigger clear protocols for more intensive cardiometabolic risk modification strategies. Mental Health Professionals' expectations 
should be raised as to what prescribing ought to achieve with a prescription being viewed as only small part of a package of care that includes education, monitoring and health promotion interventions.

Focused interventions for those receiving more intensive mental health care may present a timely and higher yield opportunity to alter outcomes particularly the heightened risk of mortality and morbidity from circulatory and respiratory disease seen within 1 year of discharge (Hoang et al. 2011). Inpatient environments provide an ideal opportunity to intensively support individuals in smoking cessation. Where smoking is the greatest predictor of early death in schizophrenia (Dickerson et al. 2013) and smoking cessation interventions are efficacious in reducing smoking rates (Banham \& Gilbody, 2010), the argument for large scale roll-out of smoke-free services with smoking cessation services is clear. These may not only lead to changes in smoking habits, but also possibly even reduced violence within the inpatient setting (Velasco et al. 1996; Lawn \& Pols, 2005; Moss et al. 2010; Voci et al. 2010; Hehir et al. 2013; Lawn \& Campion, 2013).

Where individuals have developed diabetes, hypertension, dyslipidaemia and obesity there is a need for more assertive strategies beyond screening to implement effective treatments and to bridge large diagnosis and treatment gaps. There are many established effective treatments for these conditions but actual treatment rates within the SMI population remain worryingly low (Nasrallah et al. 2006; Hippisley-Cox et al. 2007; Crawford et al. 2014). The UK Lester Cardio-metabolic resource (Lester et al. 2012) based on Australian work (Curtis et al. 2012) is designed to support the shift in focus from screening to intervening and is a welcome contribution to the management of physical health in SMI. It does not however provide a solution to the questions of where, how and by whom interventions should be delivered.

\section{Alterative care models}

Primary care is best equipped to deliver treatments for these conditions but current models of care including for example short consultation times and reliance on variable degrees of patient self-management are frequently inadequate to meet the needs of many with SMI. There is a role for individual needs assessment and more flexible models to deliver equitable services and change the current status quo (Druss et al. 2001; Vreeland, 2007; Crompton et al. 2010). These could include longer consultation times, adjunct nurse led appointments to support education and shared clinics with mental health workers to support individuals whose engagement may be affected by positive, cognitive or negative symptoms associated with psychosis.

New technologies such as smart phone applications, activity monitoring devices or online physical health selfmanagement programmes should be evaluated as potential additional tools to support health literacy and promote ownership over physical and mental health in service users (NHS Confederation, 2013; Meyer et al. 2014).

There is a key role for mental health services to work with primary care providers and consider their reciprocal roles in facilitating access to services, engagement with treatment plans and service models that address the individual needs of service users. Service planners should consider investment in technology, including shared information platforms that cross mental and physical health care systems to facilitate better information transfer. Such investment is predicted to lead to long-term efficiency savings alongside improvements in quality in care.

\section{Other physical illness challenges}

To date the primary focus in the literature has been on cardiometabolic disease. Although an appropriate priority, there are other important and legitimate targets for health services and researchers in the field of physical health in SMI. Treatment gaps, poorer disease outcomes and barriers to physical health exist across the full spectrum of diseases including for example respiratory illness, cancer, renal disease and dentistry (Kisely et al. 2005; Leucht et al. 2007; Lawrence et al. 2013).

Further investment in the evidence base to identify and target disease areas with increased prevalence in $\mathrm{SMI}$ is required. There is a need to look across the spectrum of disease and associated services and consider adjustments that facilitate equitable access to all physical heath services. The challenge here lies not only in considering more flexible care pathways, but also in reversing the separate approaches to body and mind. Training and educating physical and mental health workers on the frequency, nature and impact of physical and mental health co-morbidity is an essential step in reducing prevalent diagnostic overshadowing (MaP, 2009) and closing the diagnosis, treatment and outcome gaps.

\section{Recommendations for future strategies and collaborators}

The degree of the mortality gap is well established and we propose as others have done (Thornicroft, 2011) that now is the time to invest and focus on a research agenda that builds upon this descriptive data and 
focuses on the interventions and systems through which this issue can be addressed.

To successfully address the problem this research community must engage and foster focused collaboration with a wide range of fields across public health, mental health, primary care, physical health, dentistry, social care, health economics, law, stigma studies, education and health services research. Without a clear statement of purpose and branding of this field we suggest it unlikely that such collaborations can develop and attract the required research funding to move this agenda beyond vision statements about parity of esteem to actual improvements in outcomes.

Lessons can be learnt from Liaison Psychiatry and primary care mental health in common mental disorder where clear inroads have been made in bridging the knowledge gap between providers. Primary care is a key stakeholder in addressing these challenges but engagement will likely not come without incentivisation or consideration from mental health services as to where we could better support primary care services. The development of shared education programmes between primary care and mental health trainees presents an exciting opportunity for the next generation of clinicians to better understand each other's needs and reduce structural barriers.

Beyond nurturing of networks and relationships, financially incentivised frameworks also offer a strategy to lever change. In the UK, both primary care and mental health services have used such incentive frameworks in the form of Commissioning for Quality and Innovation (CQUIN; NHSEngland, 2015) and Quality Outcomes Framework (QOF; DoH, 2013) targets. Although welcome in signalling the importance of physical health in SMI to the respective health providers, the degree to which these influence longer term outcomes or become embedded in routine clinical care once financial incentivisation is removed is unclear. Furthermore, the UK experience of replication of near parallel monitoring requirements in both primary care and mental health reveal the disconnect between these sectors and highlight the role for more sophisticated payment mechanisms with levers such as joint outcomes frameworks and integrated commissioning guides.

These issues reinforce the need for mental health to take a key leadership role in defining the research agenda and engaging other disciplines in a focused, strategic and multidisciplinary response. Despite the robust arguments from equity to address the mortality gap, more powerful arguments to both attract research funding and also facilitate changes in service provision will come from economics. Despite the prediction that the costs to society of this co-morbidity, disability and mortality gap are substantial, the accumulating evidence on the economic case for action is still modest
(Mangalore \& Knapp, 2007; Lemmi, 2014). A commitment from research groups is required to ensure that studies are accompanied by appropriately powered and robust economic analyses.

A final but important hurdle for both clinicians and researchers to address is risk of selection bias and perpetuation of exclusion of certain service users with SMI from clinical trials. A frequent clinical consequence of relapse of SMI is loss of capacity, including capacity to consent to trial inclusion. This presents a significant risk that trial results will not be valid when transferred to reallife settings and perpetuates barriers to access of effective interventions for those with SMI. This lends support to the role of pragmatic or naturalistic study designs to accumulate more evidence for this underserved population.

The difficulties we have in addressing the issue of capacity are reflected not only in research but in routine clinical practice. In this paper, we have highlighted an ongoing delay in acknowledgement of the degree of risk that physical illness presents to service users in entering the profession's consciousness. The perspectives and values we hold in organisational cultures have a direct impact on the care we deliver to patients. There is a serious need to consider with service users where institutional attitudes, values and associated behaviours may be further impacting on a perpetuation of the mortality gap. Where for example as a profession we have tacitly supported smoking, accepted weight gain as a consequence of treatment or held ideas that no real gains will be made in changing illness behaviours (such as reduced motivation or reduced activity) we communicate values about those we provide care for.

Research has already clearly revealed that we have underestimated the potential gains to those with SMI of physical health interventions. Difficult but important questions regarding the role of capacity and the stage, degree and ways we could intervene in supporting service users in better physical health are outstanding. Despite institutional memory and related concerns about restrictive practice or enforced monitoring of physical health for those with SMI there is a need to hold these conversations where capacity is compromised and to actively engage those with SMI, their family, advocates and carers in them. Without these conversations and an on-going commitment to challenge the attitudes and values embedded in our practice, we will fail to fully address the most fundamental strategy in dealing with physical health in SMI, our own institutional stigma.

\section{Acknowledgements}

FG and BS receive funding support from the National Institute for Health Research Collaboration for Leadership in Applied Health Research \& Care 
Funding scheme. The views expressed in this publication are those of the author(s) and not necessarily those of the NHS, the National Institute for Health Research or the Department of Health. Fiona Gaughran has received honoraria for lectures and advisory/consultation work for Lundbeck, Roche, Sunovion and Otsuka and has research funded by a NHS Innovations/Janssen-Cilag award. She has a family member with professional links to GSK and Lilly.

\section{Financial Support}

This research received no specific grant from any funding agency, commercial or not-for-profit sectors.

\section{Conflict of interest}

None.

\section{References}

ADA (2004). Consensus development conference on antipsychotic drugs and obesity and diabetes. Journal of Clinical Psychiatry 65, 267-272.

Bak M, Fransen A, Janssen J, van Os J, Drukker M (2014). Almost all antipsychotics result in weight gain: a meta-analysis. PLoS ONE 9, e94112.

Banham L, Gilbody S (2010). Smoking cessation in severe mental illness: what works? Addiction 105, 1176-1189.

Barry VW, Baruth M, Beets MW, Durstine JL, Liu J, Blair SN (2014). Fitness vs. fatness on all-cause mortality: a meta-analysis. Progress in Cardiovascular Diseases 56, 382-390.

Caemmerer J, Correll CU, Maayan L (2012). Acute and maintenance effects of non-pharmacologic interventions for antipsychotic associated weight gain and metabolic abnormalities: a meta-analytic comparison of randomized controlled trials. Schizophrenia Research 140, 159-168.

Chang CK, Hayes RD, Perera G, Broadbent MT, Fernandes AC, Lee WE, Hotopf M, Stewart R (2011). Life expectancy at birth for people with serious mental illness and other major disorders from a secondary mental health care case register in London. PLoS ONE 6, e19590.

Citrome L, Yeomans D (2005). Do guidelines for severe mental illness promote physical health and well-being? Journal of Psychopharmacology 19, 102-109.

Comission DR (2005). Equal Treatment: Closing the Gap. A Formal Investigation into Physical Health Inequalities Experienced by People with Learning Disabilities and/or Mental Health Problems. DRC: London.

Cook BL, Wayne GF, Kafali EN, Liu Z, Shu C, Flores M (2014). Trends in smoking among adults with mental illness and association between mental health treatment and smoking cessation. JAMA 311, 172-182.

Correll CU, Detraux J, De Lepeleire J, De Hert M (2015). Effects of antipsychotics, antidepressants and mood stabilizers on risk for physical diseases in people with schizophrenia, depression and bipolar disorder. World Psychiatry 14, 119-136.

Crawford MJ, Jayakumar S, Lemmey SJ, Zalewska K, Patel MX, Cooper SJ, Shiers D (2014). Assessment and treatment of physical health problems among people with schizophrenia: national cross-sectional study. British Journal of Psychiatry 205, 473-477.

Crompton D, Groves A, McGrath J (2010). What can we do to reduce the burden of avoidable deaths in those with serious mental illness? Epidemiologia e Psichiatria Sociale 19, 4-7.

Curtis J, Newall HD, Samaras K (2012). The heart of the matter: cardiometabolic care in youth with psychosis. Early Interv Psychiatry 6, 347-353.

Daumit GL, Dickerson FB, Wang NY, Dalcin A, Jerome GJ, Anderson CA, Young DR, Frick KD, Yu A, Gennusa JV III, Oefinger M, Crum RM, Charleston J, Casagrande SS, Guallar E, Goldberg RW, Campbell LM, Appel LJ (2013). A behavioral weight-loss intervention in persons with serious mental illness. New England Journal of Medicine 368, 1594-1602.

De Hert M, Dekker JM, Wood D, Kahl KG, Holt RI, Moller HJ (2009). Cardiovascular disease and diabetes in people with severe mental illness position statement from the European Psychiatric Association (EPA), supported by the European Association for the Study of Diabetes (EASD) and the European Society of Cardiology (ESC). European Psychiatry 24, 412-424.

De Hert M, Correll CU, Bobes J, Cetkovich-Bakmas M, Cohen D, Asai I, Detraux J, Gautam S, Moller HJ, Ndetei DM, Newcomer JW, Uwakwe R, Leucht S (2011). Physical illness in patients with severe mental disorders. I. Prevalence, impact of medications and disparities in health care. World Psychiatry 10, 52-77.

De Hert M, Detraux J, van Winkel R, Yu W, Correll CU (2012). Metabolic and cardiovascular adverse effects associated with antipsychotic drugs. Nature Reviews: Endocrinology 8, 114-126.

Dickerson F, Stallings CR, Origoni AE, Vaughan C, Khushalani S, Schroeder J, Yolken RH (2013). Cigarette smoking among persons with schizophrenia or bipolar disorder in routine clinical settings, 1999-2011. Psychiatric Services 64, 44-50.

Dixon L, Postrado L, Delahanty J, Fischer PJ, Lehman A (1999). The association of medical comorbidity in schizophrenia with poor physical and mental health. Journal of Nervous and Mental Disease 187, 496-502.

Dixon L, Goldberg R, Lehman A, McNary S (2001). The impact of health status on work, symptoms, and functional outcomes in severe mental illness. Journal of Nervous and Mental Disease 189, 17-23.

DoH (2013). The NHS Outcomes Framework 2014/15. The Department of Health: London.

Druss BG (2007). Improving medical care for persons with serious mental illness: challenges and solutions. Journal of Clinical Psychiatry 68(Suppl. 4), 40-44.

Druss BG, Bradford WD, Rosenheck RA, Radford MJ, Krumholz HM (2001). Quality of medical care and excess mortality in older patients with mental disorders. Archives of General Psychiatry 58, 565-572. 
Fleischhacker WW, Cetkovich-Bakmas M, De Hert M, Hennekens CH, Lambert M, Leucht S, Maj M, McIntyre RS, Naber D, Newcomer JW, Olfson M, Osby U, Sartorius N, Lieberman JA (2008). Comorbid somatic illnesses in patients with severe mental disorders: clinical, policy, and research challenges. Journal of Clinical Psychiatry 69, 514-519.

Fleischhacker WW, Heikkinen ME, Olie JP, Landsberg W, Dewaele P, McQuade RD, Loze JY, Hennicken D, Kerselaers W (2010). Effects of adjunctive treatment with aripiprazole on body weight and clinical efficacy in schizophrenia patients treated with clozapine: a randomized, double-blind, placebo-controlled trial. International Journal of Neuropsychopharmacology 13, 1115-1125.

Harris EC, Barraclough B (1998). Excess mortality of mental disorder. British Journal of Psychiatry 173, 11-53.

Hehir AM, Indig D, Prosser S, Archer VA (2013). Implementation of a smoke-free policy in a high secure mental health inpatient facility: staff survey to describe experience and attitudes. BMC Public Health 13, 315.

Hippisley-Cox J, Parker C, Coupland C, Vinogradova Y (2007). Inequalities in the primary care of patients with coronary heart disease and serious mental health problems: a cross-sectional study. Heart 93, 1256-1262.

Hoang U, Stewart R, Goldacre MJ (2011). Mortality after hospital discharge for people with schizophrenia or bipolar disorder: retrospective study of linked English hospital episode statistics, 1999-2006. BMJ 343, d5422.

Kisely S, Smith M, Lawrence D, Maaten S (2005). Mortality in individuals who have had psychiatric treatment: population-based study in Nova Scotia. British Journal of Psychiatry 187, 552-558.

Kisely S, Campbell LA, Wang Y (2009). Treatment of ischaemic heart disease and stroke in individuals with psychosis under universal healthcare. British Journal of Psychiatry 195, 545-550.

Lamb HR (1989). Improving our public mental health systems. Archives of General Psychiatry 46, 743-744.

Lambert TJ, Velakoulis D, Pantelis C (2003). Medical comorbidity in schizophrenia. Medical Journal of Australia 178(Suppl.), S67-S70.

Laursen TM, Munk-Olsen T, Agerbo E, Gasse C, Mortensen PB (2009). Somatic hospital contacts, invasive cardiac procedures, and mortality from heart disease in patients with severe mental disorder. Archives of General Psychiatry 66, 713-720.

Lawn S, Campion J (2013). Achieving smoke-free mental health services: lessons from the past decade of implementation research. International Journal of Environmental Research and Public Health 10, 4224-4244.

Lawn S, Pols R (2005). Smoking bans in psychiatric inpatient settings? A review of the research. Australian and New Zealand Journal of Psychiatry 39, 866-885.

Lawrence D, Kisely S (2010). Inequalities in healthcare provision for people with severe mental illness. Journal of Psychopharmacology 24, 61-68.

Lawrence D, Hancock KJ, Kisely S (2013). The gap in life expectancy from preventable physical illness in psychiatric patients in Western Australia: retrospective analysis of population based registers. BMJ 346, f2539.
Lemmi KM (2014). The Economic Case for Better Mental Health. In Annual Report of the Chief Medical Officer 2014.

Lester HSD, Rafi I, Cooper S, Holt R (2012). Positive cardiometabolic health resource: an intervention framework for patients with psychosis on antipsychotic medication. In Royal College of Psychiatrists. London. Retrieved 1 February 2016 from http://www.rcpsych.ac.uk/ pdf/LesterUKAdaptation2014updateA5booklet_portrait_ version.pdf

Leucht SBT, Henderson J, Maj M, Sartorius N (2007). Physical Illness and Schizophrenia: A Review of the Evidence. Cambridge University Press: Cambridge.

Mangalore R, Knapp M (2007). Cost of schizophrenia in England. Journal of Mental Health Policy and Economics 10, 23-41.

MaP HP (2009). Mental and physical health charter, bridging the gap between mental and physical health. The mental and physical health platform. Retrieved 1 February 2016 from https://www.idf.org/sites/default/files/Mental\% 2520and\%2520Physical\%2520Health\%2520Charter\%2520\%2520FINAL.pdf

Meyer N, Dobson R, MacCabe J (2014). Remote monitoring of sleep-wake activity as an early warning sign in psychosis: a novel, exploratory approach. In Early Intervention in Psychiatry Meeting Abstract

Mitchell AJ, De Hert M (2015). Promotion of physical health in persons with schizophrenia: can we prevent cardiometabolic problems before they begin? Acta Psychiatrica Scandinavica 132, 83-85.

Mitchell AJ, Malone D, Doebbeling CC (2009). Quality of medical care for people with and without comorbid mental illness and substance misuse: systematic review of comparative studies. British Journal of Psychiatry 194, 491-499.

Mitchell AJ, Vancampfort D, De Herdt A, Yu W, De Hert M (2013). Is the prevalence of metabolic syndrome and metabolic abnormalities increased in early schizophrenia? A comparative meta-analysis of first episode, untreated and treated patients. Schizophrenia Bulletin 39, 295-305.

Mitchell AJ, Pereira IE, Yadegarfar M, Pepereke S, Mugadza V, Stubbs B (2014). Breast cancer screening in women with mental illness: comparative meta-analysis of mammography uptake. British Journal of Psychiatry 205, 428-435.

Moore S, Shiers D, Daly B, Mitchell AJ, Gaughran F (2015). Promoting physical health for people with schizophrenia by reducing disparities in medical and dental care. Acta Psychiatrica Scandinavica 132, 109-121.

Moss TG, Weinberger AH, Vessicchio JC, Mancuso V, Cushing SJ, Pett M, Kitchen K, Selby P, George TP (2010). A tobacco reconceptualization in psychiatry: toward the development of tobacco-free psychiatric facilities. American Journal on Addictions 19, 293-311.

Nasrallah HA, Meyer JM, Goff DC, McEvoy JP, Davis SM, Stroup TS, Lieberman JA (2006). Low rates of treatment for hypertension, dyslipidemia and diabetes in schizophrenia: data from the CATIE schizophrenia trial sample at baseline. Schizophrenia Research 86, 15-22.

NHS Confederation (2013). E-mental health: what's all the fuss about. Retrieved 1 February 2016 from http://www. nhsconfed.org/resources/2013/01/e-mental-health-whatsall-the-fuss-about 
NHSEngland (2015). Commissioning for Quality and Innovation (CQUIN) Guidance for 2015/16.

NICE (2014). Clinical guideline 178: Psychosis and schizophrenia in adults: treatment and management.

Osborn DP, Nazareth I, King MB (2006). Risk for coronary heart disease in people with severe mental illness: cross-sectional comparative study in primary care. British Journal of Psychiatry 188, 271-277.

Oslo ÖÖ (1936). Mortality in Norwegian mental hospitals from 1916 to 1933. Acta Psychiatrica Scandinavica 11, 323-356. RCPsych (2009). Physical Health in Mental Health. Royal College of Psychiatrists: London.

RCPsych (2013). Whole-person Care: from Rhetoric to Reality. Royal College of Psychiatrists: London.

Rosenbaum S, Tiedemann A, Sherrington C, Curtis J, Ward PB (2014). Physical activity interventions for people with mental illness: a systematic review and meta-analysis. Journal of Clinical Psychiatry 75, 964-974.

Rubin RR, Peyrot M, Wang NY, Coughlin JW, Jerome GJ, Fitzpatrick SL, Bennett WL, Dalcin A, Daumit G, Durkin N, Chang YT, Yeh HC, Louis TA, Appel LJ (2013). Patient-reported outcomes in the practice-based opportunities for weight reduction (POWER) trial. Quality of Life Research 22, 2389-2398.

Scott D, Platania-Phung C, Happell B (2012). Quality of care for cardiovascular disease and diabetes amongst individuals with serious mental illness and those using antipsychotic medications. Journal for Healthcare Quality 34, 15-21.

Shiers D, Jones PB, Field S (2009). Early intervention in psychosis: keeping the body in mind. British Journal of General Practice 59, 395-396.

Shore JH (1996). Psychiatry at a crossroad: our role in primary care. American Journal of Psychiatry 153, 1398-1403.

Singer RB (2001). The first mortality follow-up study: the 1841 Report of William Farr (physician) on the mortality of lunatics. Journal of Insurance Medicine 33, 298-309.

Smith DJ, Langan J, McLean G, Guthrie B, Mercer SW (2013). Schizophrenia is associated with excess multiple physical-health comorbidities but low levels of recorded cardiovascular disease in primary care: cross-sectional study. BMJ Open 3(4), pii: e002808.

Thornicroft G (2011). Physical health disparities and mental illness: the scandal of premature mortality. British Journal of Psychiatry 199, 441-442.
Tiihonen J, Lonnqvist J, Wahlbeck K, Klaukka T, Niskanen L, Tanskanen A, Haukka J (2009). 11-year follow-up of mortality in patients with schizophrenia: a populationbased cohort study (FIN11 study). Lancet 374, 620-627.

Vancampfort D, Rosenbaum S, Ward PB, Stubbs B (2015a). Exercise improves cardiorespiratory fitness in people with schizophrenia: a systematic review and meta-analysis. Schizophrenia Research 169, 453-457.

Vancampfort D, Stubbs B, Mitchell AJ, De Hert M, Wampers M, Ward PB, Rosenbaum S, Correll CU (2015b). Risk of metabolic syndrome and its components in people with schizophrenia and related psychotic disorders, bipolar disorder and major depressive disorder: a systematic review and meta-analysis. World Psychiatry 14, 339-347.

Vancampfort D, Rosenbaum S, Schuch FB, Ward PB, Probst M, Stubbs B (2015c). Prevalence and predictors of treatment dropout from physical activity interventions in schizophrenia: a meta-analysis. General Hospital Psychiatry. doi: 10.1016/j.genhosppsych.2015.11.008.

Velasco J, Eells TD, Anderson R, Head M, Ryabik B, Mount R, Lippmann S (1996). A two-year follow-up on the effects of a smoking ban in an inpatient psychiatric service. Psychiatric Services 47, 869-871.

Voci S, Bondy S, Zawertailo L, Walker L, George TP, Selby P (2010). Impact of a smoke-free policy in a large psychiatric hospital on staff attitudes and patient behavior. General Hospital Psychiatry 32, 623-630.

Vreeland B (2007). Bridging the gap between mental and physical health: a multidisciplinary approach. Journal of Clinical Psychiatry 68(Suppl. 4), 26-33.

Walker ER, McGee RE, Druss BG (2015). Mortality in mental disorders and global disease burden implications: a systematic review and meta-analysis. JAMA Psychiatry 72, 334-341.

Ward MC, White DT, Druss BG (2015). A meta-review of lifestyle interventions for cardiovascular risk factors in the general medical population: lessons for individuals with serious mental illness. Journal of Clinical Psychiatry 76, e477-e486.

Wilkinson DG (1979). Psychiatrists with blinkers. Lancet 2, 252-253.

Wu RR, Zhao JP, Guo XF, He YQ, Fang MS, Guo WB, Chen JD, Li LH (2008). Metformin addition attenuates olanzapine-induced weight gain in drug-naive first-episode schizophrenia patients: a double-blind, placebo-controlled study. American Journal of Psychiatry 165, 352-358. 